\title{
1 Decision Support Systems for Real-World High-Speed Rail Planning
}

2 By Ana Laura Costa, Ph.D. ${ }^{(1)(*)}$, Maria da Conceição Cunha, Ph.D. ${ }^{(2)}$, Paulo A. L. F. Coelho, Ph.D. ${ }^{(3)}$, and

3 Herbert H. Einstein, Sc.D., F. ASCE ${ }^{(4)}$

4 (1) Researcher, Department of Civil Engineering, University of Coimbra, alcosta@dec.uc.pt, ${ }^{(*)}$ corresponding

5 author

6 (2) Professor, Department of Civil Engineering, University of Coimbra, mccunha@dec.uc.pt

7 (3) Assistant Professor, Department of Civil Engineering, University of Coimbra, pac@dec.uc.pt

8 (4) Professor, Department of Civil and Environmental Engineering, Massachusetts Institute of Technology,

9 einstein@mit.edu

\section{ABSTRACT}

13 The selection of the macro-location of new High-Speed Rail (HSR) systems during the planning stage affects

14 the associated infrastructure costs. The process is influenced by the complex interaction between the HSR alignment, the technical solutions and the characteristics of the deployment site, subject to layout restrictions. Decision-support systems for the optimization of the HSR alignment are developed for addressing the requirements of large and complex real projects. The formulation includes costs, geometric constraints, connection requirements and consideration of natural barriers such as protected land-use and bodies of water, ubiquitous in real projects. The Simulated Annealing Algorithm is implemented to address challenges of real problems and solve the optimization model. The approach is applied to a Portuguese HSR case. The solution obtained optimizes its alignment by minimizing the construction costs, consistent with existing projects worldwide, and complying with location, geometry and land-use restrictions. The approach is not case-specific and can be used to systematically study trade-off opportunities and support decision-making in similar planning problems. Alternative solutions can be generated based on different judgments on the trade-offs. Keywords:

High-speed rail; Macro-location planning; Decision-support systems; Simulated Annealing Algorithm; 
31 When planning new High-Speed Rail (HSR) systems, initial decisions are made concerning the infrastructure macro-location. Project specifics such as the type of traffic (passenger-only, freight or mixed) and the design speed imply different geometric requirements (CEN 2002; EC 2008) and track displacement limits (RTRI 2007), which influence the HSR configuration. These project specifics coupled with site characteristics, such as elevation, geology, geotechnical behavior, population density or climate, determine the technical solutions to implement. Furthermore, the HSR must consider land-use in protected areas and crossing bodies of water. As the site characteristics can vary significantly along a HSR line, so can the technical solutions adopted, which relate to varying construction costs. Campos and de Rus (2009) compiled data from HSR projects in Europe, South Korea and Taiwan observing that the construction cost per km (without planning and land expropriation costs) varied between $€ 4.7$ million and $€ 65.8$ million (in 2005 euros). Moreover, the macro-location of HSR systems constrains subsequent optimization processes for specific infrastructure sections and the location of stations that are crucial for the HSR success (Brons et al. 2009). As a result, it is extremely difficult to obtain the HSR configuration yielding the most value, particularly in the planning stage, in which large areas and significantly different configurations can be considered.

Complex decisions need to be made, and large investments are necessary, when defining rail alignments and selecting the number and locations of stations along the rail line. As discussed in the literature, the optimization of the location of rail stations and the optimization of rail alignments can lead to significant savings in investment costs and operation costs of rail systems while also satisfying other objectives (Kang et al. 2014). Extensive research exists on the optimization of rail alignments and the location of stations (Jha et al. 2007; Kang et al. 2014; Repolho et al. 2013; Samanta and Jha 2011). However, there are intertwined aspects of HSR rail planning (Repolho et al. 2013) and a fully-integrated approach, which can also consider optional intermediate stations in-between fixed terminal ones, was not found in the literature.

Addressing these issues, Costa et al. (2013) proposed an optimization model for HSR standard planning conditions (SPC), i.e. under, ordinary operating conditions prevailing within the lifetime of the infrastructure. The objective function intends to minimize construction costs and optimize geometric layout, land-use and the inclusion of intermediate stations, providing a systematic approach to trade-off opportunities between such factors. A user-friendly computational tool was developed to solve the proposed SPC model. The tool implemented the Simulated Annealing Algorithm (SAA) (Kirkpatrick et al. 1983) for solving the model, 
implemented for solving the highway alignment optimization problem but limitations have been identified in addressing model- and/or problem specifics (Kang et al. 2012). Within local search techniques, the SAA implementation produced good results in solving similar optimization problems (Angulo et al. 2012; Marques et al. 2015; Zeferino et al. 2012).

The optimization model by Costa et al. (2013) represented interacting factors affecting the HSR planning in the conceptual stage. The conception of the model and the implementation and calibration of the solving technique were illustrated for a simple and synthetic case-study. However, transitioning to real-world complexity raises challenges, both for the model formulation and the implementation of the solving technique (Maier et al. 2014) that this paper addresses. Larger problem size and complex interacting factors, typical of real-world decision-making, develop additional difficulties that decision support systems developed for simple problems do not consider. The optimization model and the solving technique need to deal with intricacies that abound in real projects, and the sheer size of the problems may increase the computation burden beyond tolerable bounds. While synthetic case-studies are valuable for proof of concept, the refinements of the approach discussed here are required for tackling the complexities of real-world problems. This paper develops decision support systems for real-world high-speed rail planning problems and its contributions address:

- Natural barriers to the infrastructure

- Effects of layout safety requirements

- Infrastructure costs

Based on the conceptual and operational frameworks developed, the capabilities of the approach are illustrated for the specific case of the Lisbon-Oporto HSR planning problem. This HSR aims at linking Lisbon, Coimbra and Porto with a passenger-dedicated double-track HSR line (Fig.1). The HSR layout configuration is represented by linear sections that connect a set of sequential 3D points in space. A discretization mesh for the case-study area, with a grid of $2 \mathrm{~km}$ in plan view ( $\mathrm{x}$ and $\mathrm{y}$ directions) and $10 \mathrm{~m}$ in elevation, defines the set of all permissible node positions from which a limited number is selected to be connected by the HSR line. The study considers that the connection of the cities Aveiro and Leiria is optional, depending on trade-offs with additional construction and operation costs. The input maps are obtained from geographic information systems.

\section{The Simulated Annealing Algorithm}

87 The Simulated Annealing Algorithm (SAA) traces its origins to the annealing process of materials to low energy states and is credited to Kirkpatrick et al. (1983) who applied the Metropolis concepts (Metropolis et al. 1953) to solve the travelling salesman problem. Costa et al. (2013) overview the algorithm and comprehensive 

algorithm starts with an initial system configuration, and neighboring configurations are tested and accepted as current configurations if they improve the value of the objective function. Worsening system configurations are also accepted as current configurations with a probability based on the Metropolis criteria, allowing the SAA to escape from local optima. The SAA is as a stochastic technique that applies a probabilistic mechanism for accepting worse solutions (Aarts et al. 1997). However, even if the algorithm is disassociated from the physical meaning, the terminology borrowed from the annealing physical process is used (Johnson et al. 1989). The probability of accepting worsening configurations decreases as the algorithm progresses (cooling) at descending values of a control parameter (temperature).

\section{Implementation of the Simulated Annealing Algorithm}

100 Based on the SAA principles, three main elements are necessary for an implementation of the algorithm: a set of

101 parameters governing the convergence of the algorithm (cooling schedule), an initial system configuration, and the procedures to generate new candidate configurations within a neighborhood structure. The initial system configuration and the generation of new candidate configurations are problem-specific. For the Lisbon-Oporto case, the HSR solutions are defined by a linear alignment connecting a set of sequential nodes. Feasibility requires that nodes representing the cities of Oporto, Coimbra and Lisbon are connected (Fig.1), and that applicable regulatory and safety requirements for geometric design and land-use restrictions are complied with.

\section{Cooling Schedule}

108 The cooling schedule of the SAA defines the finite sequence of values of the temperature (control parameter)

109 and a finite number of transitions at each temperature by specifying (Aarts et al. 1997):

$110 \quad-\quad$ an initial value of the temperature $t_{0}$;

111 a decrement function for lowering the temperature;

112 - the finite length of each homogeneous Markov chain, meaning a minimum number of iterations $n_{l}$ to be 113 performed at each temperature step;

114 - a termination criterion for the algorithm.

115 While the SAA may be applied to solve a wide range of problems, the algorithm parameters producing the best 116 solutions in each case depend on the problem solved and its size (Johnson et al. 1989). Pardalos et al. (2000) 117 discuss the difficulty of choosing a cooling schedule, as its performance for a particular problem cannot be fully 118 appreciated a priori. The choice relies on an adaptive geometric (Johnson et al. 1989), as implemented by Costa 119 et al. (2013) based on research by Cunha (1999), Cunha et al. (2009) and Johnson et al. (1989). Each $k^{\text {th }}$ temperature decrease step is governed by $t_{k}=r^{k} \times t_{0}$, with a decrease rate $r$ and an initial temperature $t_{0}=-0.1$ 
$121 c\left(s_{0}\right) / \ln (a)$, where $c\left(s_{0}\right)$ is the value of the objective function of the initial configuration and $a$ is the elasticity of

122 acceptance defining the probability of accepting a worsening solution at the initial temperature step. The SAA is

123 terminated if $n_{2}$ consecutive temperature decrease steps do not improve either the optimum or the average value

124 of the HSR configurations.

\section{Initial System Configuration}

126 Different possible methodologies can be used for defining an initial system configuration such as the use of

127 heuristics (Johnson and McGeoch 1997) or an arbitrarily random or best guess configuration (Bertsimas and

128 Nohadani 2010). However, if the SAA implementation allows one to conduct the global search of the feasible

129 space, the initial configuration will not interfere with the accessibility of the search space nor interfere with the

130 quality of the final solution (Bertsimas and Nohadani 2010). Given the significant overhead computation time

131 associated with random- and heuristic- generated configurations, the case-study implements an arbitrary user-

132 specified, feasible initial HSR. Feasibility ensures compliance with the problem constraints; at this stage, they

133 are mandatory connections, land-use and geometry of the alignment, but further model developments can

134 impose additional requirements.

\section{Generation of New System Configurations}

136 Consider the definition of the discretization mesh $\Omega_{N}$ (Fig. 2) whose vertices represent the permissible 3D

137 positions for the nodes defining the HSR alignment. Obtaining a new candidate HSR alignment consists of 138 defining a new set of nodes and their respective linear sections. The neighborhood structure thus defines the maximum envelope distance at which each node can be repositioned.

140 Fig. 2a illustrates the 3D neighborhood of a current (center) node of $\Omega_{N}$ within its discretization mesh.

141 Moves are allowed to any of the adjacent nodes varying $\mathrm{x}, \mathrm{y}$ and/or $\mathrm{z}$ that define the neighborhood envelope.

142 Nodes that are mandatorily connected by the HSR (see location constraint by Costa et al. (2013)) have particular

143 neighborhood structures. In the case-study, Oporto, Coimbra and Lisbon are the mandatory nodes with a fixed 144 location and moves of any kind are disallowed.

145 The plan view of Fig. 2b shows a current configuration (formed by linear sections c1, c2 and c3) 146 connecting the mandatory start and end nodes that is perturbed into a neighboring configuration (formed by 147 linear sections $\mathrm{n} 1, \mathrm{n} 2$ and $\mathrm{n} 3$ ). The plan view of sections $\mathrm{c} 3$ and $\mathrm{n} 3$ coincides. Note that even if three148 dimensionally coincident, the construction costs of sections c3 and n3 are not necessarily equal. The technical 149 solutions adopted for the cross-section govern the construction cost and these can vary for two linear sections 150 with identical 3D alignment. For example, if a bridge is required in the new section n2, it may be extended into 151 a part of section $\mathrm{n} 3$, while sections c2 and c3 require only embankments. This emphasizes the continuity 
152 required in the cross-sections, for which the technical solution at a given point may be influenced by those required upstream and downstream of that point.

154 The generation of new candidate HSR configurations aims at allowing small rearrangements to be tested instead of profound changes. This is affected by the neighborhood structure but also by the degree of

156 freedom with which the current configuration is perturbed. In the case of the HSR optimization problem, the degree of freedom relates to the number of nodes to be randomly perturbed to generate a new alignment. If the

158 degree of freedom is too low, the algorithm can be circumscribed to part of the design space, but if the degree of freedom is too large, the algorithm engages in a random search and refrains from taking advantage of the local

160 neighborhood search properties (Jilla and Miller 2001). Based on preliminary studies for the Portuguese case, the SAA implementation perturbs two random nodes of the current HSR configuration.

\section{Addressing Real-World Complexity}

163 Additional challenges develop when aiming at solving real-world HSR planning problems. The model formulation can only realistically represent the problem if existing conditions such as crossing bodies of water and determinant construction costs are accounted for. On the other hand, the solving technique is required to address such model complexities and large datasets. The SAA is implemented based on the framework discussed in the previous section but real and complex problems require that a specific implementation be tailored. These issues are now discussed and illustrated for the specific case of the Lisbon-Oporto HSR line based on real data (RAVE 2008).

\section{$170 \quad$ Natural Barriers}

171 Land-Use

172 Different land-use areas, irregularly sized and shaped, exist in real problems. Specific areas can be protected

173 under regulatory frameworks and HSR overlay may be barred. When such areas are scattered within the search space of the problem, the ability of the SAA to perform a global search can be limited and, as a result, the ability of the algorithm of finding optimal or near-optimal solutions is compromised.

177 where protected land-use exist, in which HSR overlay is not allowed. Starting from an initial arbitrary HSR

178 (Fig. 3a), under the current procedures for generating new system configurations, an implementation of the SAA

179 results in a sub-optimal solution (Fig. 3b), as later proven. The algorithm is able to search the problem space in multiple positions relative to the smaller protected areas but larger protected areas act as barriers that the SAA 
space of the problem, hindering the algorithm convergence to global optima.

Advanced mechanisms are adopted for the generation of new candidate configurations that eliminate

184 the restrictions posed to the SAA convergence by the protected land-use. One major difficulty of the process is to account for the constraints that limit the minimum horizontal angles (proxy for radii) at intermediate nodes of

186 the HSR configurations when generating new HSR alignments. These constraints aim at ensuring a smooth change of direction in plan view, as required for the high operating speeds. Consider the example of Fig. 4, for which the current node $\mathrm{N}$ is randomly chosen to be horizontally repositioned to the right ( $\left.\mathrm{N}^{\prime}\right)$ by the spacing of the discretization mesh $\delta$. If a small area of protected land-use exists (Fig. 4a), the move to N' can successfully avoid the protected area. However, for a larger area (Fig. 4b), this repositioning distance $\left(\Delta_{1}=\delta\right)$ is not sufficient, as the candidate configuration still overlays the protected area.

The procedures adopted consist in incrementing the repositioning distance of node $\mathrm{N}$ in the original direction. Fig. $4 \mathrm{~b}$ shows that repositioning does not avoid the area unless $\Delta_{3}=\delta$. However, this shift leads to increasingly smaller, not acceptable horizontal angles at nodes $\mathrm{N}^{\prime}, \mathrm{N}-1$ and $\mathrm{N}+1$. Hence, a new candidate configuration obtained with the incremental displacement may be infeasible if not complying either with the land-use constraint or the horizontal angle constraint. In this latter case, a new tentative generation procedure is considered, which also repositions the anterior and posterior nodes. Fig. 4c shows how the alignment from Fig. $4 \mathrm{~b}$ can be successfully transposed by repositioning the nodes $\mathrm{N},(\mathrm{N}-1)$ and $(\mathrm{N}+1)$.

Given that the algorithm implementation may have to consider various shapes and sizes of protected areas, a general procedure is developed. It consists of the tentative generation of feasible HSR configurations in five sequential steps and stops when a feasible configuration is reached. It aims at defining a new candidate configuration that smoothly repositions itself in relation to the protected land-use area but can still be framed within the neighborhood principles of the SAA. The steps involve the repositioning of a number of anterior and posterior nodes in addition to the randomly chosen node $\mathrm{N}$. In each step, the displacement of $\mathrm{N}$ is incremented until a feasible configuration is found. If the displacement causes non-compliance with the horizontal angle constraint, or the repositioning distance extends beyond the search space of the problem, the current step stops and the subsequent step starts. The detailed sequence of these steps is presented below.

211 - Step 2: nodes N, (N-1), (N-2), (N+1) and (N+2) are displaced (Fig. 5a). (N-1) and (N+1) are repositioned 
212 at $2 / 3$ of the $\mathrm{N}$ displacement and $(\mathrm{N}-2)$ and $(\mathrm{N}+2)$ are repositioned at $1 / 3$, all in the same direction as $\mathrm{N}$. It

213 should be noted that the problem discretization requires all node positions to correspond to a node in $\Omega_{N}$.

214 Thus all repositioning distances are rounded to the closest multiple of $\delta$, as exemplified in Fig. 5a.

215 - Step 3: nodes $\mathrm{N},(\mathrm{N}-1),(\mathrm{N}-2),(\mathrm{N}-3),(\mathrm{N}+1),(\mathrm{N}+2)$ and $(\mathrm{N}+3)$ are displaced (Fig. 5b). $(\mathrm{N}-1)$ and $(\mathrm{N}+1)$ are 216 repositioned at $3 / 4$ of the $\mathrm{N}$ displacement, $(\mathrm{N}-2)$ and $(\mathrm{N}+2)$ are repositioned at $1 / 2$ of the $\mathrm{N}$ displacement 217 and $(\mathrm{N}-3)$ and $(\mathrm{N}+3)$ are repositioned at $1 / 4$ of the $\mathrm{N}$ displacement, all in the same direction as $\mathrm{N}$.

218 - Step 4: nodes N, (N-1), (N-2), (N-3), (N-4), (N+1), $(\mathrm{N}+2),(\mathrm{N}+3)$ and $(\mathrm{N}+4)$ are displaced. $(\mathrm{N}-1)$ and $219(\mathrm{~N}+1)$ are repositioned at $8 / 9$ of the $\mathrm{N}$ displacement, $(\mathrm{N}-2)$ and $(\mathrm{N}+2)$ are repositioned at $7 / 9$ of the $\mathrm{N}$ displacement, $(\mathrm{N}-3)$ and $(\mathrm{N}+3)$ are repositioned at 2/9 of the $\mathrm{N}$ displacement and $(\mathrm{N}-4)$ and $(\mathrm{N}+4)$ are repositioned at $1 / 9$ of the $\mathrm{N}$ displacement, all in the same direction as $\mathrm{N}$. The displacement of $\mathrm{N}$ is incremented until a feasible configuration is found, but if it causes the non-compliance with the horizontal angle constraint or the repositioning extends beyond the search space of the problem, Step 4 is abandoned. At this point, the generation of HSR candidate configurations is restarted and a new node $\mathrm{N}$ is randomly chosen to be displaced in its neighborhood (Figure 2).

\section{Bodies of Water}

227 Bodies of water such as rivers or lakes commonly affect transport infrastructure projects. In fact, establishing 228 HSR links often involves building bridges and tunnels to overcome such natural barriers. In specific cases, the bodies of water can be part of waterway routes, and navigability concerns are imposed on the construction of new infrastructure. Such is the case of the Lisbon-Oporto HSR that inevitably entails the construction of bridges or tunnels (Fig. 6). Furthermore, navigability is required in parts of the Tagus River.

To realistically represent such concerns, additional constraints are implemented in the optimization model that require bodies of water to be crossed by either bridges or tunnels. These feasibility requirements

234 further determine a minimum clearance to be observed that should be defined depending on the problem specifics and on hydrological studies. The Lisbon-Oporto case study defines a minimum clearance of $5 \mathrm{~m}$ for

236 bridges crossing water, but additional navigability clearances are considered for the Tagus River. Considering

237 the characteristics of existing bridges crossing the Tagus River, a minimum height of 70 meters should be 238 complied with for additional planned bridges (Fig. 6). This makes it possible for the SAA to perform a global search of the feasible space based on realistic solutions.

\section{Effects of Layout Safety Requirements}

241 Radii of horizontal curves of the HSR layout should be planned as large as feasible and should follow regulatory 
242 minimum values (CEN 2002). Large centrifugal acceleration of trains, increasing with increasing speed and

243 decreasing radii, intensifies the degradation of the track, passenger discomfort and, in extreme cases, favors

244 conditions leading to train derailment (Profillidis 2006). The problem formulation considers a minimum

245 horizontal angle at any intermediate angle of the HSR alignment as a proxy for the radii (see Costa et al. 2013).

246 Preliminary applications to the Lisbon-Oporto problem, however, identify problematic effects of safety

247 requirements propagating into the optimized solution found by the SAA. These effects are herein presented,

248 followed by the procedures implemented to avoid them. Fig. 7 shows the formation of a cluster near Oporto, a

249 group of three or more closely positioned nodes connected by the HSR, causing small angles between linear

250 sections without advantage to the HSR configuration. This would hardly produce an optimal or near-optimal

251 solution of a real-world HSR: the operating speed is severely limited when changing direction and that would

252 imply slow movement through clusters, which is in contrast to the fundamental principles of HSR. These are

253 geometric considerations of the problem that interfere with the SAA implementation. Node clusters have

254 underlying effects on the HSR configurations generated and tested by the algorithm that, in turn, influence the

255 quality of the solutions produced.

256 However, when solving the optimization model, it is difficult to prevent node clusters, with speed restrictions and present in the current configurations, from propagating to the generated candidate configurations. Closely positioned nodes become interlocked and moves in the neighborhood are confined by the horizontal angle feasibility. Furthermore, at the low temperature stages of the SAA, in which the probability of accepting worsening configurations is also low, the HSR configurations required for such an elimination may be rejected by producing excessively large objective function values. In fact, if a cluster forms and propagates to low temperature stages of the SAA implementation most of the moves that would reverse the cluster are either forbidden due to the horizontal angle feasibility requirements or will not be accepted because the probability of accepting worse solutions is very low. It is possible but difficult to eliminate the clusters of speed restrictions.

To prevent such problematic effects and their propagation into the optimized HSR configurations, a minimum length for the HSR linear sections is defined. This minimum length is intended to disallow candidate configurations in which the nodes are closely positioned, thus avoiding the cluster formation, but should not be so large as to compromise the SAA ability to perform a global search of the problem space.

A separate study was performed for the Lisbon-Oporto problem to identify the minimum length of the

270 linear sections that allow the use of circular curves with 4500 m radius (Fig. 8): the arc is required to be tangent at each end to the respective linear sections while having a limited external secant $\Delta$. The latter ensures that the 
272 simplified HSR representation by linear alignments optimized by the model is in fact spatially related to the real

273 HSR defined by curves and linear sections. This simplified representation of the model can capture the main

274 features concerning the plan view and longitudinal profile for the macro-location planning, however, subsequent

275 detailed studies are needed to define the circular and transition curves forming the HSR line. Based on the

276 separate study, a minimum length of $4000 \mathrm{~m}$ is considered for the linear sections forming the Lisbon-Oporto

277 HSR alignment.

\section{Infrastructure Costs}

279 HSR components, such as track, ballast or catenary exist, for which the unit construction costs do not vary significantly with the in situ characteristics. While these length-dependent costs do not add major complexities to the model, their incorporation favors shorter configurations, which real projects aim at. The optimization model developed for real problems includes these length-dependent costs that not only affect construction cost but also relate to operating considerations. The length-dependent costs, together with the costs of crossing bodies of water acting as natural barriers, with bridges or tunnels, are decisive considerations for an adequate representation of the construction costs of HSR systems.

\section{Solving Real Problems: The Case of the Lisbon-Oporto HSR}

Spatial data are input in the form of digital raster maps for the $147.4 \times 304.4 \mathrm{~km}^{2}$ study area (Fig. 1). The raster maps for protected areas, lithology and ground use (APA 2012), for bodies of water (SNIRH 2012) and for elevation are discretized in $200 \mathrm{~m}$ square geo-referenced cells. The discretization mesh, defining the feasible node positions of the HSR, is formed by a grid of $2 \mathrm{~km}$ in the plan view and $10 \mathrm{~m}$ in elevation (Fig. 2a), between elevations $-50 \mathrm{~m}$ and $1420 \mathrm{~m}$. The optimization model applied to the Lisbon-Oporto HSR case-study aims at the minimization of the value of the objective function represented in a simplified form by eq. 1 .

294 Where $\mathrm{C}_{\text {Construction }}$ is the construction cost including expropriation, earthworks (cuts and embankments), bridges, tunnels and length-dependent costs, $\mathrm{P}_{\text {HorizAngle }}$ is a penalty for adopting horizontal angles smaller than best practice design value, $\mathrm{P}_{\text {Gradient }}$ is a penalty for adopting longitudinal gradients larger than best practice design value and $\mathrm{V}_{\text {InterCities }}$ represents the added value of connecting intermediate and optional cities. geometry design, but have implications on operation. This results in the definition of two values for each geometry parameter (CEN 2002): maximum (or minimum) limit values, which are extreme but permissible values that should be used in design as infrequently as possible, and recommended values consisting of best 
302 practice geometry design. The absolute safety limits defining the feasibility of the HSR, in our case are a

303 minimum horizontal angle of $120^{\circ}$ at any intermediate node of the HSR and a maximum gradient of $35 \mathrm{~mm} / \mathrm{m}$

304 for the linear sections in the longitudinal profile. The penalties in the objective function (Costa et al. 2013) are

305 related to adopting geometry parameters that are less desirable than best practice. The recommended design

306 parameters considered in the case-study are $140^{\circ}$ and $20 \mathrm{~mm} / \mathrm{m}$. The optional connection of Aveiro and Leiria is

307 also considered through a term in the objective function. The value of connecting each city represents effects

308 such as the ability to influence ridership through increased accessibility but also possible negative effects of

309 intermediate stops causing an increase in connecting times (Repolho et al. 2013), as well as critical political

310 decisions (Levinson 2012) regarding the location of HSR stations. In addition to the geometry contraints,

311 feasibility is determined by protected areas (Fig. 3), the mandatory connection of Lisbon, Coimbra and Oporto

312 and the bridge and tunnel requirements for crossing bodies of water.

313 The SAA is implemented to solve the model and address the complexities posed by real problems. An

314 extensive study is performed to establish the cooling schedule parameters discussed earlier in the SAA

315 implementation section, analogously to the study performed by Costa et al. (2013) that compares the

316 performance of the algorithm for different values of each parameter. The cooling schedule parameter set $(a=0.9$;

$\left.317 r=0.8 ; n_{1}=5000 ; n_{2}=10\right)$ is observed to be the most effective for the Lisbon-Oporto case study. The plan view of

318 the initial configuration is shown in Fig. 3a. The best solution found by the algorithm is shown in the plan views

319 of Fig. 9: a) the land-use map, b) the main rivers map and c) the elevation map. The HSR connects Oporto,

320 Aveiro, Coimbra, Leiria and Lisbon. Note that the connection of Aveiro and Leiria is not mandatory but

321 depends on the user-specified benefit attributed to the connection of each of these cities. The present study

322 includes these in a cursory manner based on Costa et al. (2013), to illustrate the capabilities of the model.

323 Detailed studies can be performed for a comprehensive representation of the value of intermediate connections.

324 Fig. 9a shows that the proposed methodology for generating HSR candidate solutions is capable of

325 addressing the difficulties caused by protected land-use areas acting as natural barriers, which is not possible

326 with previous implementations (Fig. 3b). The mechanisms implemented allow the SAA to perform a global

327 exploration of the problem space, considering radically different configurations, which is central to the

328 effectiveness of finding optimal or near-optimal solutions of optimization problems. As a result, the objective

329 function value was reduced from 2008.2 (Fig. 3b) to 786.96 Million Euros (Fig. 9a).

330 Fig. 9a also compares the plan view of the HSR solution with the existing conventional railway

331 connecting Lisbon and Oporto. The lower operating speed considered for the design of the older conventional 
railway is compatible with smaller radii of horizontal curves that, along with detours for connecting smaller

333 towns in-between major centers, results in the sinuous plan-view shown in Fig. 9a. Apart from the horizontal

334 curvature and the connection of smaller towns, both the HSR and the conventional rail share a common corridor between Oporto and just south of Coimbra and also near Lisbon. One observes that by running adjacent to the

336 Atlantic Ocean, between Oporto and Aveiro, close to the Mondego River, south of Coimbra, and along the

337 Tagus River, next to Lisbon (Fig. 9b), both rail infrastructures take advantage of the smaller costs of building on

338 level ground (Fig. 9c). Fig. 10 shows the HSR longitudinal profile and ground elevation along the alignment,

339 with identification of tunnels and bridges. Note, however, that building embankments and cuts over alluvium, in

340 the flatter areas constituting the floodplains of the Mondego and Tagus Rivers, would produce some important

341 geotechnical concerns with implications on both the construction and operation phases. These should be further

342 studied in detail.

343 The HSR is $296.3 \mathrm{~km}$ long, and tunnels are mostly built between $\mathrm{km} 270$ and $\mathrm{km} 285$ where high 344 elevations with sharp variations impose the need for tunnels (Fig. 10). Bridges are built along the alignment due to both topography and crossing bodies of water, ensuring a minimum of $5 \mathrm{~m}$ clearance for the latter. The HSR

346 does not cross the Tagus River and thus its navigability is not affected. While bodies of water may be crossed by either bridges or tunnels, bridges are usually less expensive and are favored by the optimized solution when technically viable.

The plan views of Fig. 9 also show that two pronounced curves exist in the HSR alignment around

350 Coimbra. This increases the HSR length by deviating from a more direct straight path south of Coimbra and increases the length-dependent construction costs. This sinuosity, however is associated with trade-offs between several cost factors constituting the objective function, namely, the curvature penalty (see expression (1)), on the one hand but avoiding, on the other hand, major construction in urban areas (Fig. 11), with larger expropriation costs. It also reduces the construction costs of bridges and tunnels by running on the flatter ground parallel to the Mondego River (Fig. 9b and c).

The resulting construction costs in 2008 Euros are shown in Fig. 12. It should be noted that the construction costs differ from the objective function value (eq. 1), the latter also representing geometry considerations and the value of intermediate connections. The aggregate total construction cost of the HSR is $€ 1,658.50$ million. Earthworks are the largest partial construction cost representing $27 \%$ of the total, followed by bridges (26\%), length-dependent costs (21\%), tunnels (17\%) and the cost of land expropriation (9\%). The fact 
that length-dependent costs are larger than those of tunnels and expropriation shows how length-dependent costs can exert a significant influence on the analysis.

An analysis is performed to investigate how the derived costs compare with costs of existing HSR projects. Campos and de Rus (2009) discuss upper and lower bounds of average construction costs per kilometer

365 (in 2005 Euros) of new and operating HSR lines around the world. The cost bounds vary significantly with in situ conditions and project specifics, even when singular projects of considerable complexity are excluded: in Italy costs vary between 14 and 65.8 million Euros (lines under construction), in France between 4.7 and 18.8 million Euros (lines in service) and in Spain between 7.8 and 20 million Euros (lines in service). An analogous comparative cost for the case-study solution can be estimated at 5.61 million per km (in 2005 Euros), which is close to cost average values of existing HSR projects (Campos and de Rus 2009). While the aim of the decisionsupport systems is to derive optimal HSR configurations based on interrelated factors additional to the construction costs, budget limitations exist. In this framework, the construction costs from the Lisbon-Oporto HSR are consistent with the costs observed in real-world projects.

\section{Conclusions}

375 Planning for High Speed Rail (HSR) infrastructure macro-location requires that a complex interrelation of spatially variable factors are accounted for. These include regulatory frameworks for infrastructure and land-use, the economic and social value of the intermediate connections and the investments required for building and operating HSR infrastructure. An integrated consideration of such elements is paramount in planning for infrastructure. Previous conceptual studies proposed an optimization model for such an approach and sound results were obtained for a simple and synthetic case-study. However, solving real problems emphasizes the need for addressing additional complexities, but also introduces additional concerns to be modeled.

This paper proposes a decision-support system for real-world problems. The definition of the HSR configuration and the connection of intermediate locations are intertwined aspects of the problem that the model can address for macro-location decisions. The challenges posed by natural barriers including protected land-use and crossing bodies of water are addressed. Mechanisms are implemented that enable one to conduct a comprehensive search of the problem solutions irrespective of existing land-use barriers. Crossing bodies of water is established with the construction of bridges and tunnels and navigability concerns are introduced where necessary. Moreover, the optimization model formulation includes length-dependent costs and the costs of crossing bodies of water that influence the infrastructure alignment and are essential for representing real HSR projects. 
obtained show the approach's ability to represent the characteristics of real problems and obtain valuable

solutions. Overall it is shown how the HSR solution optimizes its alignment by minimizing construction costs,

which are consistent with existing HSR projects worldwide, while addressing land-use, geometry and location

issues. The approach can be used to systematically study trade-off opportunities and support decision-making.

Alternative solutions can be generated based on different judgments on the trade-offs. The approach is not case-

specific and can be applied to other HSR and similar transportation planning problems. Further developments

may consider additional technical solutions and should incorporate operating conditions.

399

400

401

402

403

404

405

406

407

408

409

410

411

412

413

414

415

416

417

418

419

420

421

422

423

424

425

426

427

428

429

\section{Acknowledgements}

The research was carried out at the University of Coimbra and the Massachusetts Institute of Technology with the financial support of the Government of Portugal through the MIT|Portugal Program and FCT doctoral grant (Grant No. SFRH/BD/43012/2008), co-financed by the European Social Fund (ESF) through POPH - Programa Operacional Potencial Humano. The authors also acknowledge the access to preliminary studies provided by former RAVE - Rede Ferroviária de Alta Velocidade, S.A.

\section{References}

Aarts, E., Korst, J., and Van Laarhoven, P. J. M. (1997). “Simulated Annealing.” Local Search in Combinatorial Optimization, John Wiley \& Sons, Inc., New York, NY, USA, 91-120.

Angulo, E., Castillo, E., Garcia-Rodenas, R., and Sanchez-Vizcaino, J. (2012). "Determining highway corridors.” J. Transp. Eng., 10.1061/(ASCE)TE.1943-5436.0000361.

APA (Portuguese Environmental Agency). (2012). "Environmental Atlas.”, $<$ http://sniamb.apambiente.pt/webatlas/>.

Bertsimas, D., and Nohadani, O. (2010). "Robust optimization with simulated annealing." J. Global Optim., 48(2), 323-334.

Brons, M., Givoni, M., and Rietveld, P. (2009). “Access to railway stations and its potential in increasing rail use." Transport. Res. A-Pol., 43(2), 136-149.

Campos, J., and de Rus, G. (2009). "Some stylized facts about high-speed rail: A review of HSR experiences around the world." Transport Policy, 16(1), 19-28.

CEN (European Committee for Standardization). (2002). "Railway application - Track alignment design parameters - Track gauges 1435 mm and wider - Part 1: Plain Line.” ENV 13803-1, Brussels.

Costa, A. L., Cunha, C., Coelho, P. a L. F., and Einstein, H. H. (2013). “Solving High-Speed Rail Planning with the Simulated Annealing Algorithm." J. Transp. Eng, 10.1061/(ASCE)TE.1943-5436.0000542, 635-642.

Cunha, M. C. (1999). “On solving aquifer management problems with simulated annealing algorithms.” Water Resour. Manage., 13(3), 153-169.

Cunha, M. C., Pinheiro, L., Zeferino, J., Antunes, A., and Afonso, P. (2009). “Optimization Model for Integrated Regional Wastewater Systems Planning.” J. Water Resour. Plann. Manage., 10.1061/(ASCE)0733-9496(2009)135:1(23).

EC (European Commission). (2008). “Commission Decision of 20 December 2007 concerning a technical specification for interoperability relating to the infrastructure sub-system of the trans-European high-speed rail system.” Off. J. Eur. Union, <http://eur-lex.europa.eu/> (Nov. 30, 2011). 
430

431

432

433

434

435

436

437

438

439

440

441

442

443

444

445

446

447

448

449

450

451

452

453

454

455

456

457

458

459

460

461

462

463

464

465

466

467

468

469

470

471

472

473

474

Jha, M. K., Schonfeld, P., and Samanta, S. (2007). "Optimizing rail transit routes with genetic algorithms and geographic information system.” J. Urban Plann. Dev., 133(3), 161-171., 10.1061/(ASCE)07339488(2007)133:3(161)

Jilla, C. D., and Miller, D. W. (2001). "Assessing the performance of a heuristic simulated annealing algorithm for the design of distributed satellite systems." Acta Astron., 48(5-12), 529-543.

Johnson, D. S., Aragon, C. R., MCGeoch, L. A., and Schevon, C. (1989). “Optimization by Simulated Annealing - An Experimental Evaluation . Part 1. Graph Partitioning.” Oper. Res., 37(6), 865-892.

Johnson, D. S., and McGeoch, L. A. (1997). “The traveling salesman problem: a case-study.” Local Search in Combinatorial Optimization, John Wiley \& Sons Ltd, New York, NY, USA, 215-310.

Kang, M. W., Jha, M. K., and Schonfeld, P. (2012). "Applicability of highway alignment optimization models." Transport. Res. C-Emer., 21(1), 257-286.

Kang, M. W., Jha, M. K., and Buddharaju, R. (2014). "Rail Transit Route Optimization Model for Rail Infrastructure Planning and Design: Case Study of Saint Andrews, Scotland.” J. Transp. Eng, 140(1), 1-11. doi: 10.1061/(ASCE)TE.1943-5436.0000445

Kirkpatrick, S., Gelatt, C. D., and Vecchi, M. P. (1983). “Optimization by simulated annealing.” Science, 220(4598), 671-680.

Van Laarhoven, P. J. M., and Aarts, E. H. L. (1987). Simulated annealing: theory and applications. Kluwer Academic Publishers Group, Dordrecht, The Netherlands.

Levinson, D. M. (2012). “Accessibility impacts of high-speed rail.” Journal of Transport Geography, 22(0), 288-291.

Maier, H. R., Kapelan, Z., Kasprzyk, J., Kollat, J., Matott, L. S., Cunha, M. C., Dandy, G. C., Gibbs, M. S., Keedwell, E., Marchi, A., Ostfeld, A., Savic, D., Solomatine, D. P., Vrugt, J. A., Zecchin, A. C., Minsker, B. S., Barbour, E. J., Kuczera, G., Pasha, F., Castelletti, A., Giuliani, M., and Reed, P. M. (2014). "Evolutionary algorithms and other metaheuristics in water resources: Current status, research challenges and future directions." Environ. Modell. Softw., 62, 271-299.

Marques, J., Cunha, M., and Savić, D. (2015). "Using Real Options in the Optimal Design of Water Distribution Networks.” J. Water Resour. Plann. Manage., 10.1061/(ASCE)WR.1943-5452.0000448.

Metropolis, N., Rosenbluth, A. W., Rosenbluth, M. N., Teller, A. H., and Teller, E. (1953). "Equation of State Calculations by Fast Computing Machines.” J. Chem. Phys., 21(6), 1087-1092.

Pardalos, P. M., Romeijn, H. E., and Tuy, H. (2000). "Recent developments and trends in global optimization." J. Comput. Appl. Math., 124(1-2), 209-228.

Profillidis, V.A. (2006). Railway management and engineering, Ashgate Publishing Co.

RAVE. (2008). "High-Speed Rail Connection between Lisbon and Oporto.” Preliminary Project Design, Lisbon, Portugal.

Repolho, H. M., Antunes, A. P., and Church, R. L. (2013). “Optimal Location of Railway Stations: The LisbonPorto High-Speed Rail Line.” Transp. Sci., 47(3), 330-343.

RTRI (Railway Research Technical Institute). (2007). Design Standards for Railway Structures - Displacement Limits, Japan.

Samanta, S., and Jha, M. K. (2011). "Modeling a rail transit alignment considering different objectives." Transport. Res. A-Pol., 45(1), 31-45.

SNIRH (Portuguese National Information System on Hydrological Resources). (2012). "Water Atlas”, $<$ http://geo.snirh.pt/AtlasAgua/>.

Zeferino, J. A., Cunha, M. C., and Antunes, A. P. (2012). "Robust optimization approach to regional wastewater system planning.” J. Environ. Manag., 109, 113-122. 


\section{Figure Captions List}

476 Fig. 1. Case-study area (rectangle of $\left.147.4 * 304.4 \mathrm{~km}^{2}\right)$ and cities represented by the (x;y;z) coordinates in

$477 \quad(\mathrm{~km} ; \mathrm{km} ; \mathrm{m})$.

478 Fig. 2. Generation of HSR configurations: a) 3D neighborhood of a current (center) node and b) Plan view of 479 the perturbation of a current configuration (solid line) into a neighboring candidate configuration (dashed line).

480 Fig. 3. HSR configurations overlaying the protected land-use layer map: a) initial configuration and b) best 481 configuration found by the SAA implementation according to Costa et al. (2013).

482 Fig. 4. Transposing protected land-use areas a) successfully and b) unsuccessfully; and c) with the repositioning 483 of nodes $\mathrm{N},(\mathrm{N}-1)$ and $(\mathrm{N}+1)$.

484 Fig. 5. Transposing protected land-use areas: a) step 2 and b) step 3.

485 Fig. 6. Lisbon-Oporto rivers' map with the location of the cross-section line defining the upstream limits of the 48670 meters bridge height requirement.

487 Fig. 7. Plan view showing evidence of HSR clustering, overlaying the Lisbon-Oporto land-use layer.

488 Fig. 8. Minimum length of linear sections linking nodes $i, j$ and $k$ of the HSR alignment with an horizontal angle $489 \beta_{(i, j, k)}$, for a circular curve of $4500 \mathrm{~m}$ radius and an external secant $\Delta$.

490 Fig. 9. Plan view of HSR solution overlaid on the case-study maps: a) land-use, b) rivers, c) elevation.

491 Fig. 10. HSR longitudinal profile with indication of built extension on bridges and tunnels. Vertical 492 exaggeration of $150 x$.

493 Fig. 11. HSR plan view overlaying the expropriation cost map between Aveiro and Leiria with detail of the unit 494 expropriation costs $\left(€ / \mathrm{m}^{2}\right)$ next to the Coimbra HSR station.

495 Fig. 12. Accumulated costs (in euros of 2008) along the HSR longitudinal profile: total construction costs and 496 partials for earthworks, expropriation, bridges, tunnels and length-dependent costs. 


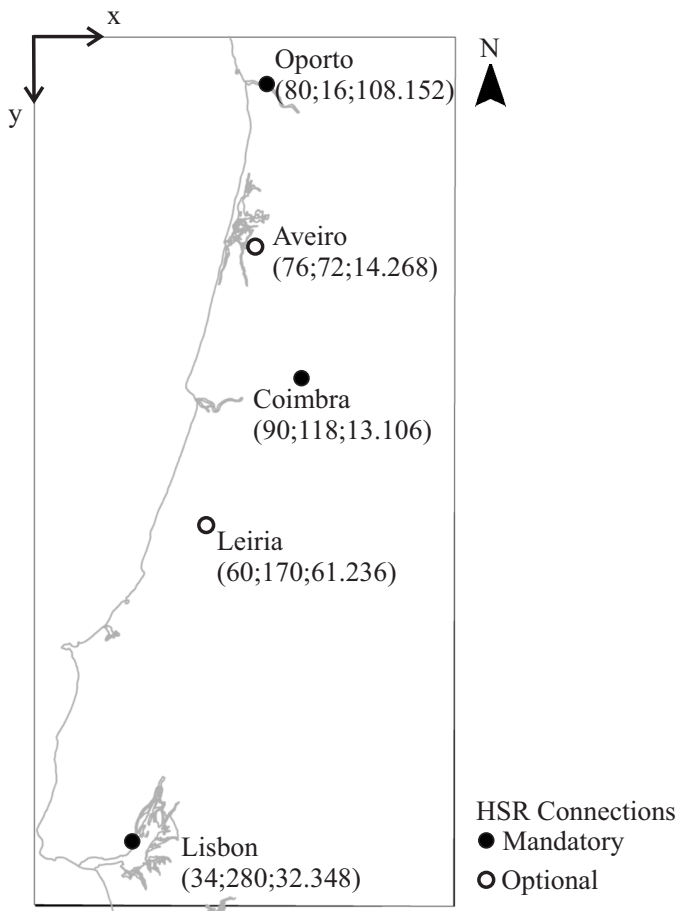

Fig. 1. Case-study area (rectangle of $\left.147.4 * 304.4 \mathrm{~km}^{2}\right)$ and cities represented by the $(\mathrm{x} ; \mathrm{y} ; \mathrm{z})$ coordinates in $(\mathrm{km} ; \mathrm{km} ; \mathrm{m})$ 


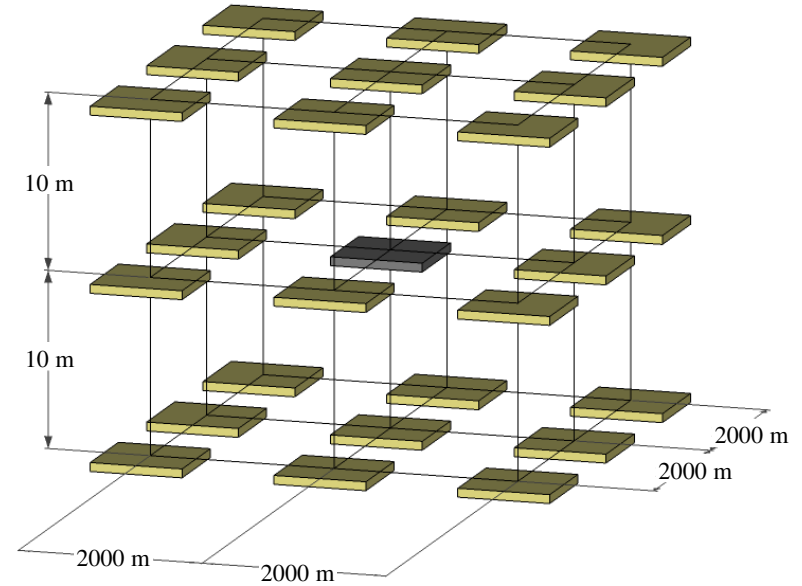

a)

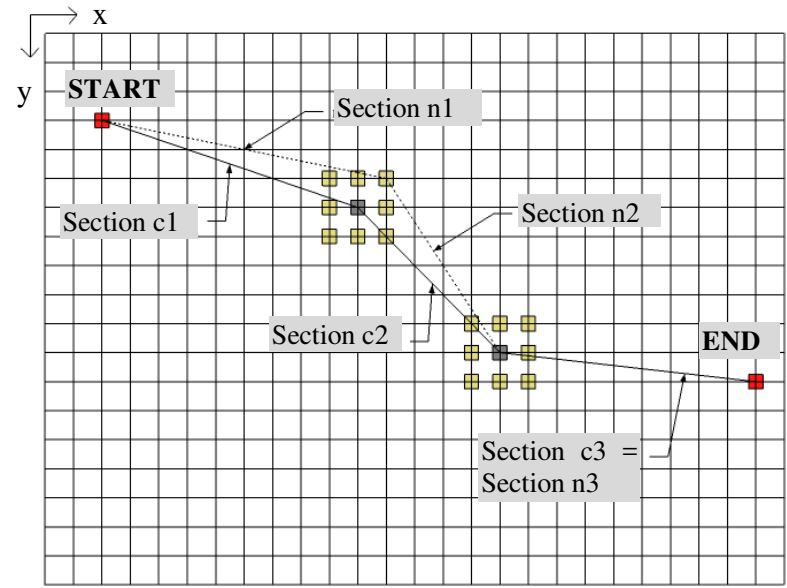

b)

Fig. 2. Generation of HSR configurations: a) 3D neighborhood of a current (center) node and b) Plan view of the perturbation of a current configuration (solid line) into a neighboring candidate configuration (dashed line). 


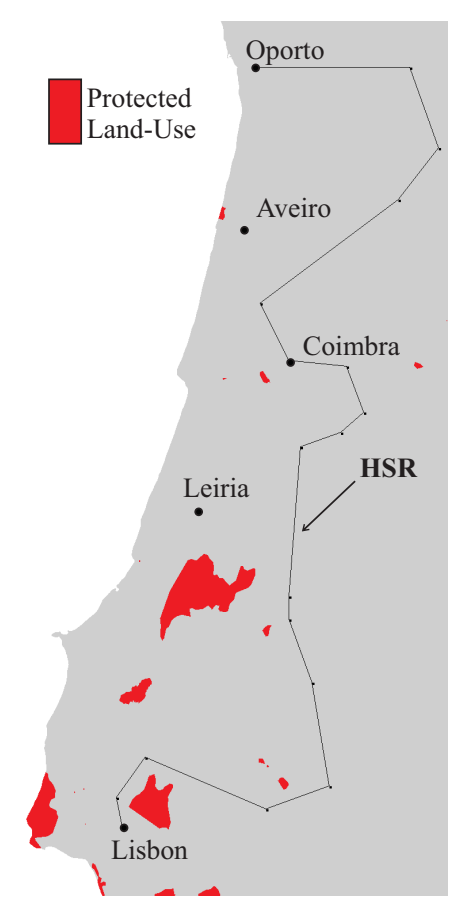

a)

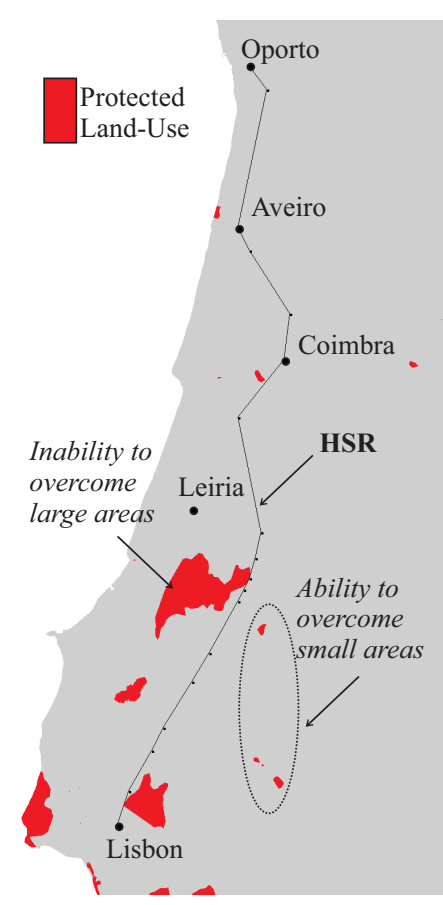

b)

Fig. 3. HSR configurations overlaying the protected land-use layer map: a) initial configuration and b) best configuration found by the SAA implementation according to Costa et al. (2013). 


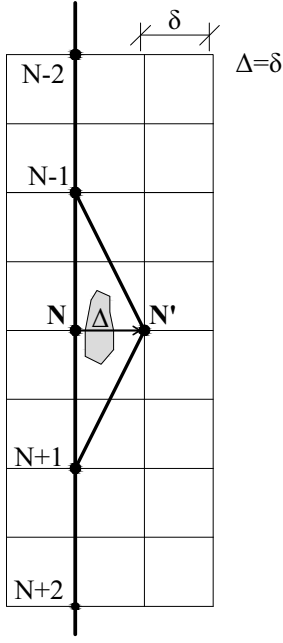

a)

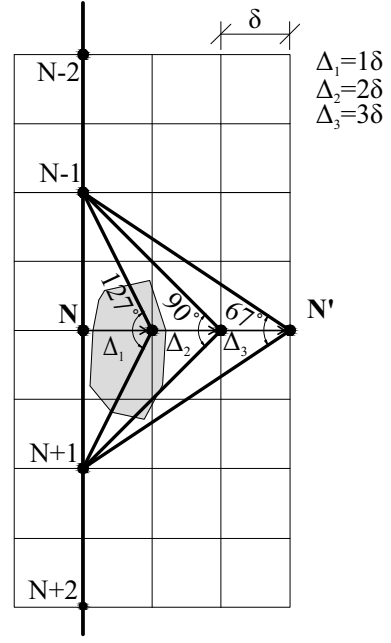

b)

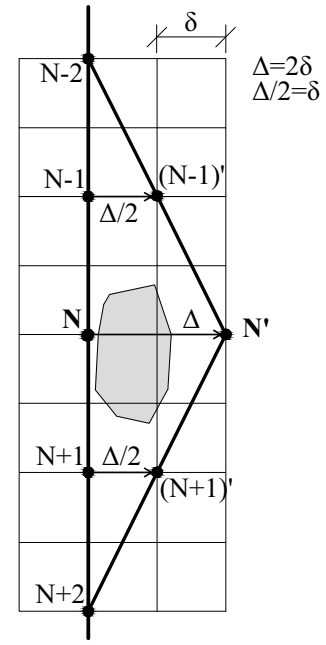

c)

Fig. 4. Transposing protected land-use areas a) successfully and b) unsuccessfully; and c) with the repositioning of nodes $\mathrm{N},(\mathrm{N}-1)$ and $(\mathrm{N}+1)$. 


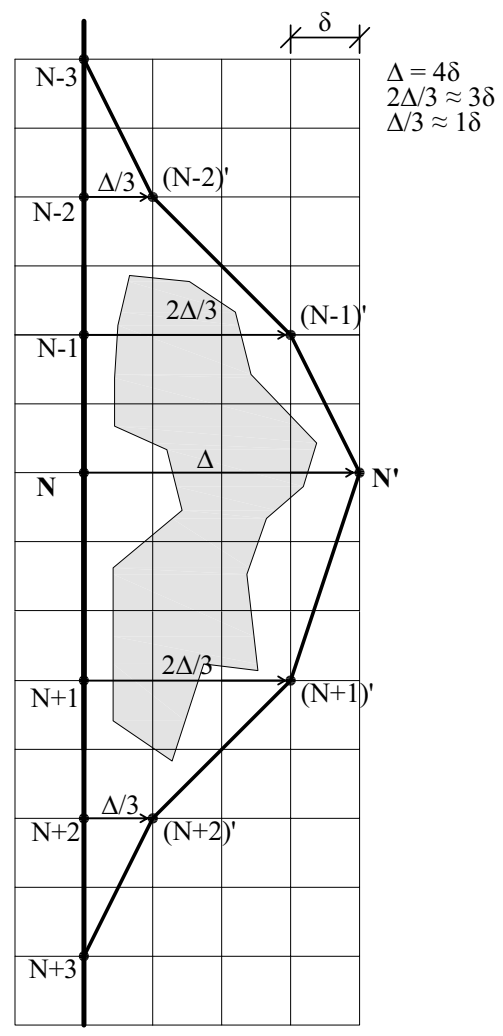

a)

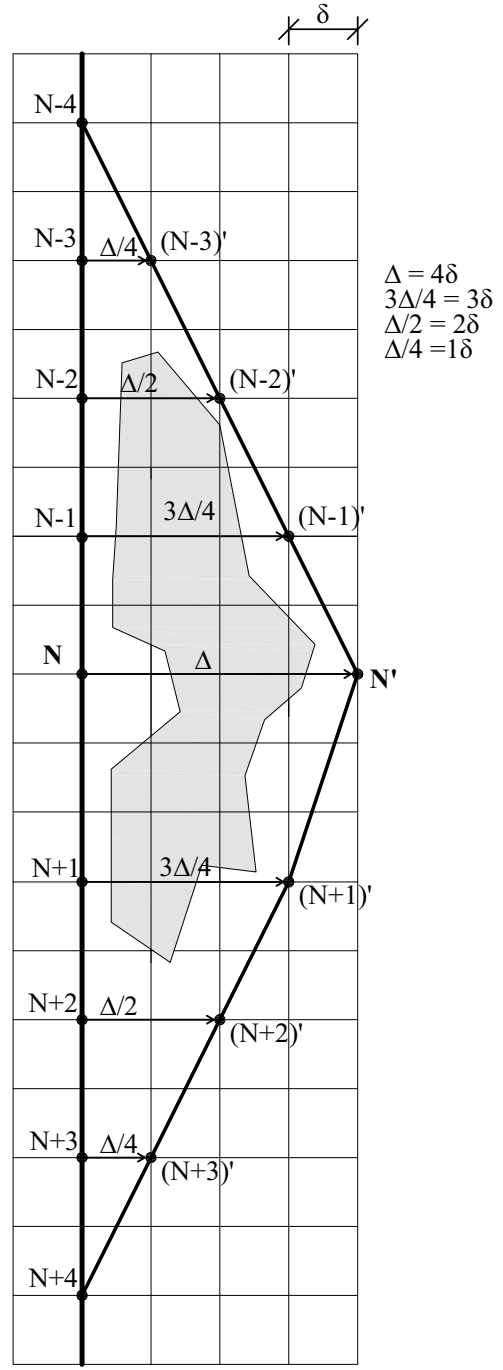

b)

Fig. 5. Transposing protected land-use areas a) step 2 and b) step 3 . 


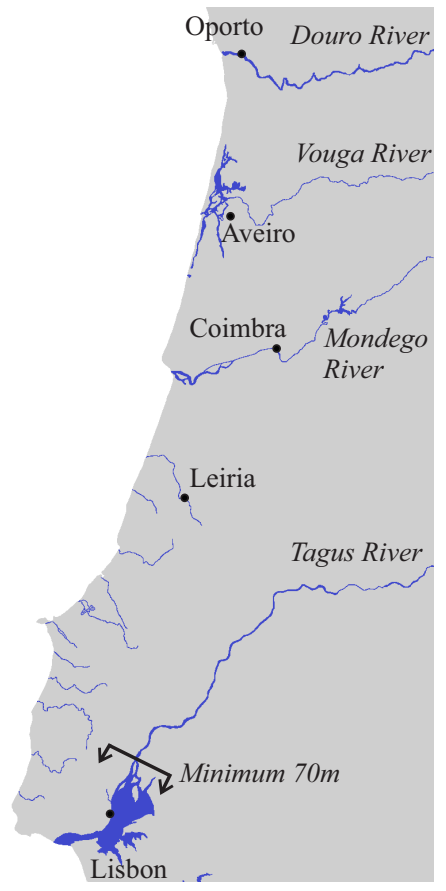

Fig. 6. Lisbon-Oporto rivers' map with the location of the cross-section line defining the upstream limits of the 70 meters bridge height requirement. 


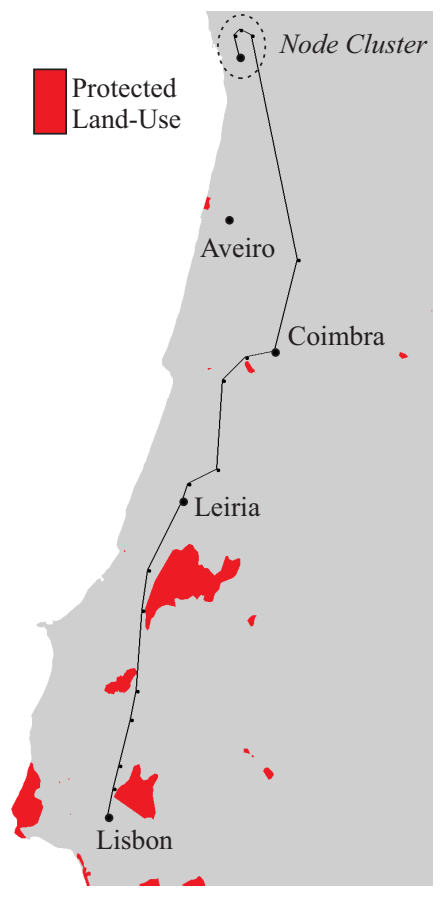

Fig. 7. Plan view showing evidence of HSR clustering, overlaying the Lisbon-Oporto land-use layer. 


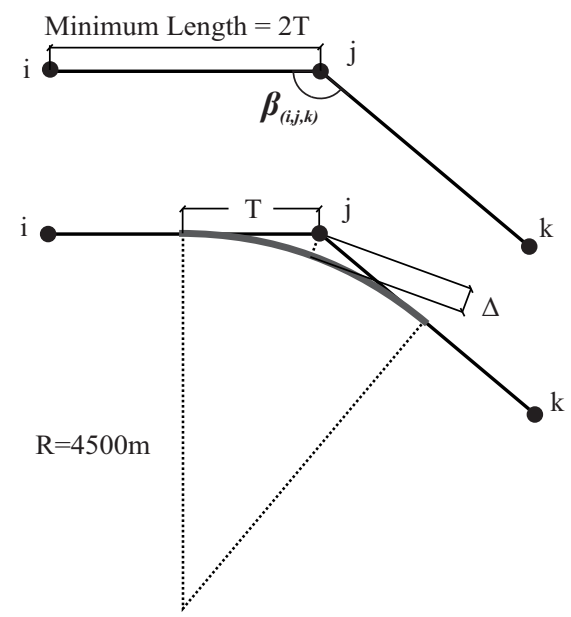

Fig. 8. Minimum length of linear sections linking nodes $i, j$ and $k$ of the HSR alignment with an horizontal angle $\beta_{(i, j, k)}$, for a circular curve of $4500 \mathrm{~m}$ radius and an external secant $\Delta$. 


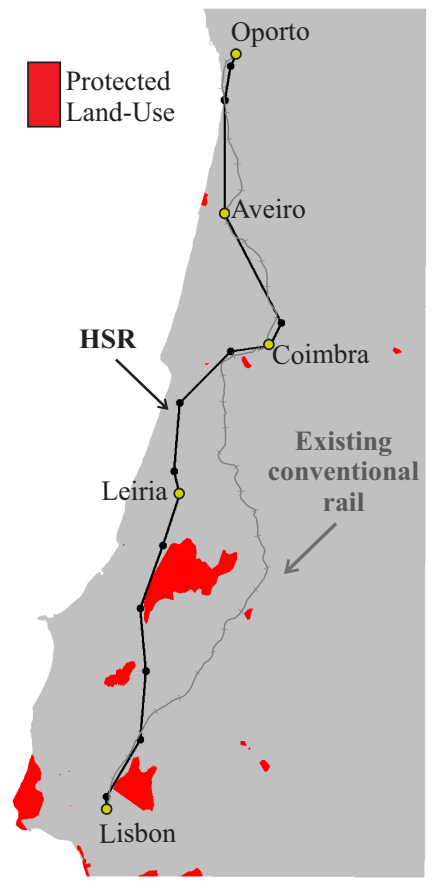

a)

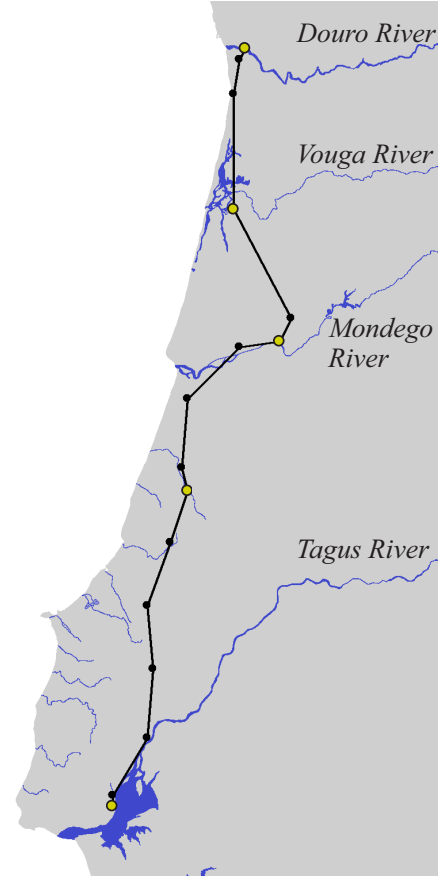

b)

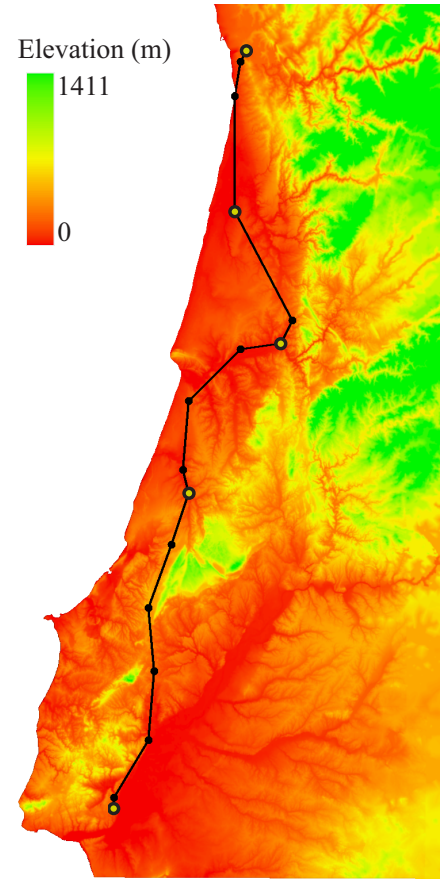

c)

Fig. 9. Plan view of HSR solution overlaid on the case-study maps: a) land-use, b) rivers, c) elevation. 


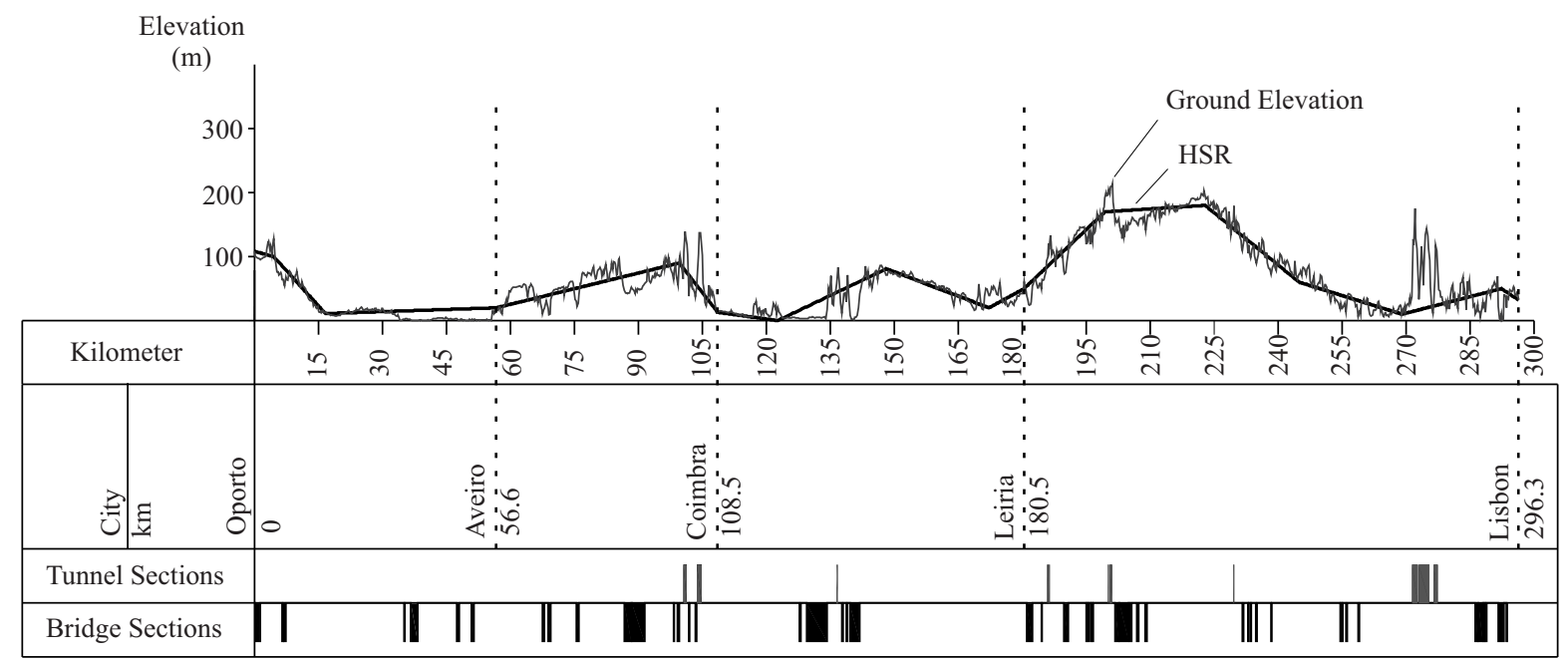

Fig. 10. HSR longitudinal profile with indication of built extension on bridges and tunnels. Vertical exaggeration of $150 \mathrm{x}$. 

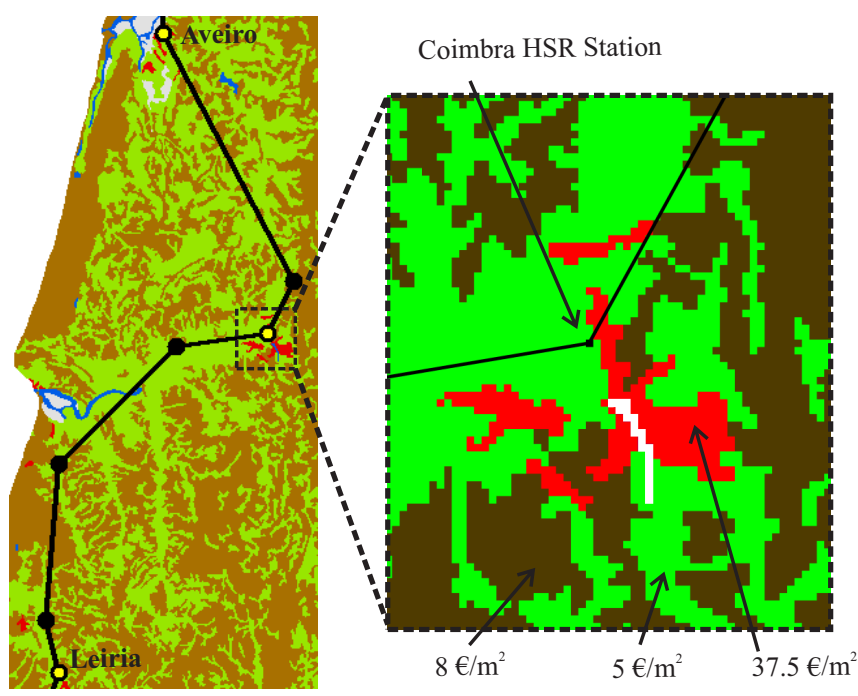

Fig. 11. HSR plan view overlaying the expropriation cost map between Aveiro and Leiria with detail of the unit expropriation costs $\left(€ / \mathrm{m}^{2}\right)$ next to the Coimbra HSR station. 


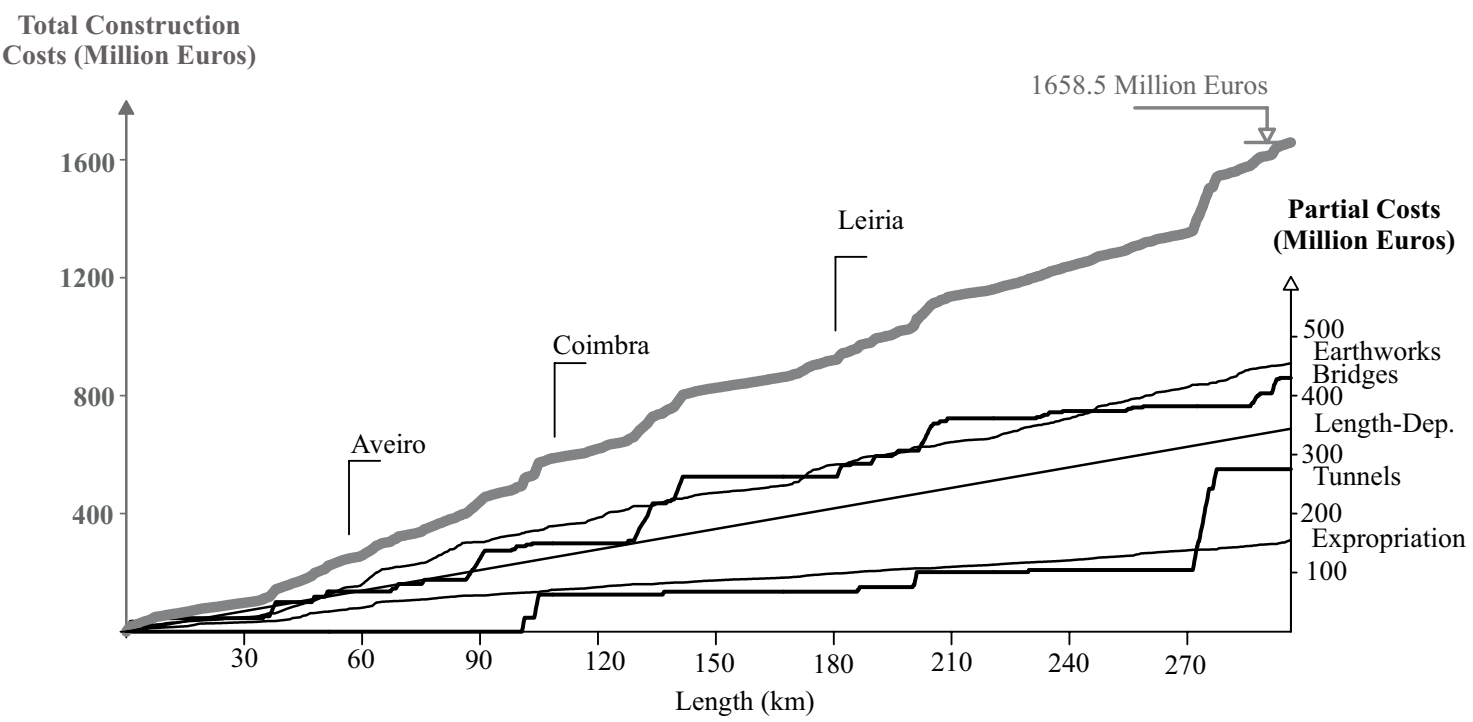

Fig. 12. Accumulated costs (in euros of 2008) along the HSR longitudinal profile: total construction costs and partials for earthworks, expropriation, bridges, tunnels and length-dependent costs. 